\title{
On a \\ Nonlinear Model of the Vibrating Membrane
}

\author{
A. Iannelli
}

Abstract. A nonlinear model describing the transversal motion of an elastic membrane is introduced and the corresponding equation is studied: An existence and uniqueness theorem for the global solution of the Cauchy-Dirichlet problem is proved.

Keywords: Mathematical models, nonlinear elasticity

AMS subject classification: Primary $35 \mathrm{~L} 20$, secondary 46 N 20, 73 C 50

\section{Introduction}

The aim of the present paper is to deduce and study a mathematical model of a homogeneous vibrating membrane of unit density, assuming transversal motion, but without making any assumptions on its smallness and considering a not necessarily linear relationship between stress and strain.

As we shall see in detail in the next section, the equation deduced and which, according to our model, represents the motion of the membrane is

$$
u_{t t}-\operatorname{div}\left[\varphi\left(\left(1+|\nabla u|^{2}\right)^{1 / 2}-\rho^{2}\right) \frac{\nabla u}{\left(1+|\nabla u|^{2}\right)^{1 / 2}}\right]-f=0
$$

where $\varphi=\varphi(s)$ represents the stress-strain law, $\rho$ is a constant depending on the initial stress with $0<\rho<1$, and $f$ is the external force. Unfortunately, it has not been possible for us to prove a global existence and uniqueness theorem for the usual Cauchy-Dirichlet problem relative to equation (1.1); hence the opportunity of modifying the model in order to make it globally "well posed".

The most obvious modification would seem to be the addition of a term $\varepsilon \Delta^{2} u$, which would take into account the fact that, in reality, the membrane can be considered as the limit of a plate, with "small" bending moment, represented by the term $\varepsilon \Delta^{2} u$. This modification does not however seem to be satisfactory, since the "modified" equation, being of parabolic type, is characterized by an infinite velocity of propagation of perturbations. We have therefore chosen a different approximation, which takes into account the fact that the sections of the membrane possess a moment of inertia which,

A. Iannelli: Politecnico di Milano, Dip. di Matematica, P.zza Leonardo da Vinci $32,{ }^{-} 20133$ Milano, Italy 
being obviously very small, is normally neglected, but which gives rise to a kinetic energy produced by their rotation. This approximation maintains the finite velocity of propagation of perturbations.

The "approximate" model we shall consider corresponds therefore to the equation

$$
u_{\imath}-\operatorname{div}\left[\varphi\left(\left(1+|\nabla u|^{2}\right)^{1 / 2}-\rho^{2}\right) \frac{\nabla u}{\left(1+|\nabla u|^{2}\right)^{1 / 2}}\right]-\gamma \Delta u_{\imath t}-f=0
$$

where $\gamma=I E$, with $I$ the moment of inertia of the sections and $E$ the Young modulus. It is obvious that, if $I=0$ and $|\nabla u|^{2}$ is "negligeable", equation (1.2) reduces to the classical equation of the vibrating membrane.

For the sake of simplicity, we shall, in what follows, assume a linear stress-strain relationship, i.e. $\varphi(s)=K s$ with $K>0$ Hooke's constant. Hence, denoting by $\Omega$ the domain of the plane covered by the membrane, we shall study the equation

$$
u_{t t}-K \Delta u+K \rho^{2} \operatorname{div}\left(\frac{\nabla u}{\left(1+|\nabla u|^{2}\right)^{1 / 2}}\right)-\gamma \Delta u_{t t}-f=0
$$

with the initial and boundary conditions

$$
\left.\begin{array}{r}
u\left(0, x_{1}, x_{2}\right)=\alpha\left(x_{1}, x_{2}\right) \\
u_{t}\left(0, x_{1}, x_{2}\right)=\beta\left(x_{1}, x_{2}\right)
\end{array}\right\} \quad\left(\left(x_{1}, x_{2}\right) \in \Omega\right)
$$

and

$$
u\left(t, x_{1}, x_{2}\right)=0 \quad\left(\left(x_{1}, x_{2}\right) \in \partial \Omega, 0 \leq t \leq T\right) .
$$

The outline of the paper is as follows. We deduce the mathematical model and give the basic definitions in Sections 2 and 3, respectively. Section 4 is devoted to the discussion of the existence problem (1.3) - (1.5), whereas in Section 5 the uniqueness is proved.

Remark 1.1. The results obtained can easily be extended to nonlinear laws, under the following physically natural assumptions on the function $\varphi$ (see also [4]):

- $\varphi \in C^{1}[0, \infty)$, with $\varphi(0)=0$ and $0<\varphi^{\prime} \leq k$.

- $c_{1} s^{\gamma}-N_{1} \leq \varphi(s) \leq c_{2} s^{\gamma}+N_{2}$ with $0<\gamma \leq 1$, where $c_{1}, c_{2}$ and $N_{1}, N_{2}$ are positive constants.

Hooke's law corresponds evidently to the special case $\gamma=1$, while, in general, the behaviour of elastic materials corresponds to values $\gamma \leq 1$ (i.e. the stress grows asymptotically at most linearly with the strain). 


\section{Deduction of the model}

Consider in the $\mathbf{x}=\left(x_{1}, x_{2}\right)$ plane a square membrane $U$ of unit surface, subject to a tension $T_{0} \geq 0$ and assume that, when $T_{0}$ vanishes, $U$ reduces to a square $U_{0}$ with sides of length $l \leq 1$. Let us stretch $U_{0}$ to a square $S$ of side $s>l$; the tension $V$ is then linked to $S$ by the relationship

$$
V=\varphi\left(s^{2}-l^{2}\right) \text {. }
$$

Consider now another square membrane $U_{\lambda}$ of the same material, which, subject to the same tension $T_{0}$, has sides of length $\lambda$ and let $\varphi_{\lambda}$ denote the stress-strain relationship relative to $U_{\lambda}$. Bearing in mind that now the tension $V$ induces an increase in the length of the sides of the squares from $s$ to $\lambda s$ and from $l$ to $\lambda l$, we have

$$
V=\varphi\left(s^{2}-l^{2}\right)=\varphi_{\lambda}\left(\lambda^{2}\left(s^{2}-l^{2}\right)\right) \quad \Longrightarrow \quad \varphi_{\lambda}\left(s^{2}-l^{2}\right)=\varphi\left(\frac{s^{2}-l^{2}}{\lambda^{2}}\right) \text {. }
$$

Let now $\Omega$ be a homogeneous membrane of unit density stretched on the $\mathbf{x}=\left(x_{1}, x_{2}\right)$ plane and fixed at the boundary $\partial \Omega$, and denote by $u(t, \mathbf{x})$ the (transversal) elongation of the point $\mathbf{x}$ at the time $t$. Consider then a square of sides $\Delta x_{1}$ and $\Delta x_{2}$ (with $\left.\Delta x_{1}=\Delta x_{2}\right)$ included in $\Omega$ and assume that, in the absence of tension, the sides of this square reduce to $\rho \Delta x_{1}$ and $\rho \Delta x_{2} \quad(0<\rho<1)$. The (approximate) potential energy of the element $\Delta x_{1} \Delta x_{2}$ when the membrane is deformed according to the function $u(t, \mathbf{x})$ is then given by

$$
\mathcal{E}_{1,2}=\int_{\rho^{2} \Delta x_{1} \Delta x_{2}}^{\left(1+|\nabla u|^{2}\right)^{1 / 2} \Delta x_{1} \Delta x_{2}} V(\sigma) d \sigma
$$

where $\nabla u$ is calculated at a point of the square $\Delta x_{1} \Delta x_{2}$. Bearing in mind (2.2), we have then

$$
\mathcal{E}_{1,2}=\int_{\rho^{2} \Delta x_{1} \Delta x_{2}}^{\left(1+|\nabla u|^{2}\right)^{1 / 2} \Delta x_{1} \Delta x_{2}} \varphi\left(\frac{\sigma}{\Delta x_{1} \Delta x_{2}}-\rho^{2}\right) d \sigma .
$$

Hence, the (approximate) potential energy of the membrane is given by

$$
\widetilde{\mathcal{E}}=\sum \mathcal{E}_{1,2}
$$

where the sum is extended to all the squares $\Delta x_{1} \Delta x_{2} \subset \Omega$. Letting $\Delta x_{1}$ and $\Delta x_{2}$ tend to zero, the potential energy of the membrane corresponding to the function $u(t, x)$ is therefore

$$
\mathcal{E}(u)=\int_{\Omega} \int_{\rho^{2} d x_{1} d x_{2}}^{\left(1+|\nabla u|^{2}\right)^{1 / 2} d x_{1} d x_{2}} \varphi\left(\frac{\sigma}{d x_{1} d x_{2}}-\rho^{2}\right) d \sigma d \mathbf{x}
$$

while the Hamiltonian associated to the motion of the membrane is given by (1)

$$
\begin{aligned}
\mathcal{H}(u)= & \int_{0}^{T}\left(-\mathcal{E}(u(t))+\int_{\Omega} \frac{1}{2} u_{t}^{2}(t ; \mathbf{x}) d \mathbf{x}\right. \\
& \left.+\int_{\Omega} \frac{1}{2} I E\left|\nabla u_{t}(t, \mathbf{x})\right|^{2} d \mathbf{x}+\int_{\Omega} f(t, \mathbf{x}) u(t, \mathbf{x}) d \mathbf{x}\right) d t
\end{aligned}
$$

(1) In view of the fact, already observed in the introduction, that the energy due to the rotation of the sections is small compared to the kinetic and potential energies, the term corresponding to the rotational energy has been linearized. 
where $f$ is the external force normal to the $\mathbf{x}$ plane.

Assuming that $u(t, \mathbf{x})=0$ when $\mathbf{x}$ belongs to $\partial \Omega$ and denoting by $h$ an arbitrary function belonging to $C^{1}([0, T] \times \Omega)$, with $h(t, \mathbf{x})=0$ when $\mathbf{x}$ belongs to $\partial \Omega$ and when $t=0$ and $t=T$, the equation of motion of the membrane in the time interval $(0, T)$ is given by

$$
\left.\frac{\partial}{\partial \varepsilon} \mathcal{H}(u+\varepsilon h)\right|_{\varepsilon=0}=0
$$

that is

$$
\int_{0}^{T} \int_{\Omega}\left\{u_{t} h_{t}-\varphi\left(\left(1+|\nabla u|^{2}\right)^{\frac{1}{2}}-\rho^{2}\right) \frac{\nabla u \nabla h}{\left(1+|\nabla u|^{2}\right)^{\frac{1}{2}}}+I E \nabla u_{t} \nabla h_{t}+f h\right\} d \mathbf{x} d t=0 .
$$

Equation (2.9) can also be written, in strong form,

$$
u_{\imath t}-\operatorname{div}\left[\varphi\left(\left(1+|\nabla u|^{2}\right)^{\frac{1}{2}}-\rho^{2}\right) \frac{\nabla u}{\left(1+|\nabla u|^{2}\right)^{\frac{1}{2}}}\right]-I E \Delta u_{\imath t}-f=0
$$

which coincides with (1.2).

Remark 2.1. The assumption that the motion is transversal corresponds to assuming that the points of the membrane move on frictionless vertical guides. On the other hand, the motion becomes "naturally" transversal if the external force is vertical and if the area of the unstretched membrane is "small".

\section{Notations and basic definitions}

Let $\Omega \subset \mathbb{R}^{2}$ be a bounded open set with a $C^{2}$-boundary or an open convex polygon and let $Q=(0, T) \times \Omega$. Furthermore, denote by $\mathbf{b}: \mathbb{R}^{2} \rightarrow \mathbb{R}^{2}$ the vector-valued function

$$
\mathbf{b}(\mathbf{x})=\frac{\mathbf{x}}{\left(1+|\mathbf{x}|^{2}\right)^{1 / 2}} \quad\left(\mathbf{x} \in \mathbb{R}^{2}\right)
$$

We shall then say that $u$ is a solution in $Q$ of problem (1.3) - (1.5), with $f \in L^{2}(Q), \alpha \in$ $H^{2}(\Omega) \cap H_{0}^{1}(\Omega)$ and $\beta \in H^{2}(\Omega)$.if:

a) $u \in W^{1, \infty}\left(0, T ; H^{2}(\Omega) \cap H_{0}^{1}(\Omega)\right)$ and $u(0)=\alpha$,

b) $u$ satisfies a.e. in $[0, T]$ the equation

$$
\begin{array}{r}
\int_{0}^{t}\left\{-\left(u^{\prime}(\tau), h^{\prime}(\tau)\right)_{L^{2}(\Omega)}\right. \\
-k(\Delta u(\tau), h(\tau))_{L^{2}(\Omega)}+\gamma\left(\Delta u^{\prime}(\tau), h^{\prime}(\tau)\right)_{L^{2}(\Omega)} \\
\left.+k \rho^{2}(\operatorname{divb}(\nabla u(\tau)), h(\tau))_{L^{2}(\Omega)}-(f(\tau), h(\tau))_{L^{2}(\Omega)}\right\} d \tau \\
+\left(u^{\prime}(t), h(t)\right)_{L^{2}(\Omega)}-(\beta, h(0))_{L^{2}(\Omega)}-\gamma\left(\Delta u^{\prime}(t), h(t)\right)_{L^{2}(\Omega)} \\
+\gamma(\Delta \beta, h(0))_{L^{2}(\Omega)}=0
\end{array}
$$

for all $h \in H^{1}\left(0, T ; L^{2}(\Omega)\right)$. 
Remark 3.1. Since $|\mathbf{b}(\mathbf{x})| \leq 1$ for all $\mathbf{x} \in \mathbb{R}^{2}$, it follows that $\mathbf{b}(\mathbf{w}) \in L^{\infty}(Q)$ for all $\mathbf{w}=\mathbf{w}(t, \mathbf{x})$ defined a.e.

Remark 3.2. Denoting by $b_{1}$ and $b_{2}$ the components of $b$, note that $\frac{\partial b_{i}}{\partial x_{j}} \in$ $L^{\infty}\left(\mathbb{R}^{2}\right)(i, j=1,2)$. Thus, by the generalized Lagrange theorem, it follows that

$$
|\mathbf{b}(\mathbf{x})-\mathbf{b}(\mathbf{y})| \leq M|\mathbf{x}-\mathbf{y}| \quad\left(\mathbf{x}, \mathbf{y} \in \mathbb{R}^{2}\right)
$$

where $M$ is an appropriate positive constant.

Remark 3.3. If $\mathbf{v}=\left(v_{1}(x, y), v_{2}(x, y)\right) \in\left(H^{1}(\Omega)\right)^{2}$, then

$$
\operatorname{div} \mathbf{b}(\mathbf{v})=\frac{\operatorname{div} \mathbf{v}+v_{1 x} v_{2}^{2}+v_{2 y} v_{1}^{2}-v_{1} v_{2}\left(v_{1 y}+v_{2 x}\right)}{\left(1+|\mathbf{v}|^{2}\right)^{3 / 2}}
$$

and $\operatorname{div} \mathbf{b}(\mathbf{v}) \in L^{2}(\Omega)$. Moreover, obviously, if $\mathbf{v} \in\left(L^{\infty}\left(0, T ; H^{1}(\Omega)\right)\right)^{2}$, then $\operatorname{div} \mathbf{b}(\mathbf{v}) \in$ $L^{\infty}\left(0, T ; L^{2}(\Omega)\right)$.

We now introduce a smoothing operator that will be employed in the sequel. Given $\delta>0$, let $g_{\delta} \in C_{0}^{\infty}(\mathbb{R})$ be the function defined by

$$
g_{\delta}(t)= \begin{cases}\frac{\mu}{\delta^{2}} \exp \left(\frac{\delta^{2}}{\delta^{2}-t^{2}}\right) & \text { for }|t|<\delta \\ 0 & \text { for }|t| \geq \delta\end{cases}
$$

with $\mu$ such that $\int_{\mathbb{R}} g_{\delta}(t) d t=1$. Furthermore, let $u=u(t, \mathbf{x})$ be a function defined a.e. in $\mathbb{R} \times \Omega$. We shall then denote by $J_{\delta}$ the regularization operator (mollifier operating only on the variable $t$ ) defined by

$$
J_{\delta} u(t, \mathbf{x})=\int_{\mathbb{R}} g_{\delta}(t-\eta) u(\eta, \mathbf{x}) d \eta
$$

for functions $u$ such that the right-hand side of (3.5) makes sense (concerning the definition and properties of $J_{\delta}$, see [1: p. 29] and [5: p. 21]). For our purposes, it is useful to recall the following

Lemma 3.1. Let $u$ and $v$ be functions defined on $\mathbb{R} \times \Omega$ and vanishing identically outside $Q=(0, T) \times \Omega$. Then:

1) If $u \in L^{p}(Q) \quad(1 \leq p<+\infty)$, then $J_{\delta} u \in L^{p}(Q)$. Moreover, $\left\|J_{\delta} u\right\|_{L^{p}(Q)} \leq\|u\|_{L^{p}(Q)}$ and $\lim _{\delta \rightarrow 0^{+}}\left\|J_{\delta} u-u\right\|_{L^{p}(Q)}=0$.

2) If $u \in W^{1,1}(Q)$, then $\frac{\partial}{\partial x_{i}} J_{\delta} u=J_{\delta} \frac{\partial u}{\partial x_{i}} \quad(i=1,2)$ and $\frac{\partial}{\partial \imath} J_{\delta} u=J_{\delta} \frac{\partial}{\partial \imath} u$.

3) $J_{\delta}$ is selfadjoint in the sense that $\left(J_{\delta} u, v\right)_{L^{2}(Q)}=\left(u, J_{\delta} v\right)_{L^{2}(Q)}$ for all $u, v \in L^{2}(Q)$.

4) $J_{\delta} \in \mathcal{L}\left(L^{2}(Q), H^{1}\left(0, T ; L^{2}(\Omega)\right)\right)$.

Finally, throughout this paper, we shall always set $L^{2}=L^{2}(\Omega), H^{s}=H^{s}(\Omega), \ldots$ and denote by $c, c_{1}, c_{2}, \ldots$ positive constants, not necessarily the same in different contexts; moreover, for simplicity, we shall always omit the integration variable. 


\section{An existence result}

In the present section we shall prove, utilizing the classical Faedo-Galerkin scheme, that problem (1.3) - (1.5) admits at least one global solution in $Q$.

Theorem 4.1. Let $f \in L^{2}(Q), \alpha \in H^{2} \cap H_{0}^{1}$ and $\beta \in H^{2}$. Then there exists in $Q$ a solution of problem (1.3) - (1.5) in $Q$.

Proof. Let $\left\{g_{j}\right\}$ be the basis in $H_{0}^{1}$ constituted by the eigenfunctions of the operator $-\Delta$ and let $\left\{\lambda_{j}\right\}$ be the corresponding eigenvalues: $-\Delta g_{j}=\lambda_{j} g_{j}$. Setting

$$
u_{n}(t)=\sum_{j=1}^{n} \alpha_{n j}(t) g_{j}
$$

we study the system of ordinary differential equations in the unknowns $\alpha_{n j}$

$$
\begin{array}{r}
\left(u_{n}^{\prime \prime}(t), g_{j}\right)_{L^{2}}-k\left(\Delta u_{n}(t), g_{j}\right)_{L^{2}}-\gamma\left(\Delta u_{n}^{\prime \prime}(t), g_{j}\right)_{L^{2}} \\
+k \rho^{2}\left(\operatorname{divb}\left(\nabla u_{n}(t)\right), g_{j}\right)_{L^{2}}-\left(f(t), g_{j}\right)_{L^{2}}=0
\end{array} \quad(j=1,2, \ldots, n)
$$

with the initial conditions

$$
\left.\begin{array}{l}
u_{n}(0)=\Pi_{n} \alpha=\alpha_{n} \\
u_{n}^{\prime}(0)=\Pi_{n} \beta=\beta_{n}
\end{array}\right\}
$$

where $\Pi_{n}$ is the projection operator on the subspace spanned by the system $\left\{g_{1}, \ldots, g_{n}\right\}$. This system admits a local solution.

We now prove that this solution can be extended to $(0, T)$. Let us multiply (4.1) by $\lambda_{j} \alpha_{n j}^{\prime}$ and add. We obtain

$$
\begin{aligned}
&\left(u_{n}^{\prime \prime}(t), \Delta u_{n}^{\prime}(t)\right)_{L^{2}}-k\left(\Delta u_{n}(t), \Delta u_{n}^{\prime}(t)\right)-\gamma\left(\Delta u_{n}^{\prime \prime}(t), \Delta u_{n}^{\prime}(t)\right)_{L^{2}} \\
&+k \rho^{2}\left(\operatorname{div} \mathbf{b}\left(\nabla u_{n}(t)\right), \Delta u_{n}^{\prime}(t)\right)_{L^{2}}-\left(f(t), \Delta u_{n}^{\prime}(t)\right)_{L^{2}}=0
\end{aligned}
$$

or, equivalently,

$$
\begin{aligned}
& \frac{1}{2} \frac{d}{d t}\left\|\nabla u_{n}^{\prime}(t)\right\|_{L^{2}}^{2}+\frac{k}{2} \frac{d}{d t}\left\|\Delta u_{n}(t)\right\|_{L^{2}}^{2}+\frac{\gamma}{2} \frac{d}{d t}\left\|\Delta u_{n}^{\prime}(t)\right\|_{L^{2}}^{2} \\
& \quad-k \rho^{2}\left(\operatorname{div} \mathbf{b}\left(\nabla u_{n}(t)\right), \Delta u_{n}^{\prime}(t)\right)_{L^{2}}+\left(f(t), \Delta u_{n}^{\prime}(t)\right)_{L^{2}}=0
\end{aligned}
$$

Integrating (4.3) between 0 and $t \in(0, T]$, we have

$$
\begin{aligned}
&\left\|\nabla u_{n}^{\prime}(t)\right\|_{L^{2}}^{2}+k\left\|\Delta u_{n}(t)\right\|_{L^{2}}^{2}+\gamma\left\|\Delta u_{n}^{\prime}(t)\right\|_{L^{2}}^{2} \\
&= 2 k \rho^{2} \int_{0}^{t}\left(\operatorname{div} \mathrm{b}\left(\nabla u_{n}\right), \Delta u_{n}^{\prime}\right)_{L^{2}} d \tau+\left\|\nabla \beta_{n}\right\|_{L^{2}}^{2} \\
&+k\left\|\Delta \alpha_{n}\right\|_{L^{2}}^{2}+\gamma\left\|\Delta \beta_{n}\right\|_{L^{2}}^{2}-2 \int_{0}^{t}\left(f, \Delta u_{n}^{\prime}\right)_{L^{2}} d \tau
\end{aligned}
$$


On the other hand, by Remark 3.3 and [2: Theorems 2.2.2.3, 3.1.2.1, 3.2.1.2 and 4.3.1.4],

$$
\begin{aligned}
\left|\left(\operatorname{div} \mathbf{b}\left(\nabla u_{n}\right), \Delta u_{n}^{\prime}\right)_{L^{2}}\right| & \leq \int_{\Omega} \frac{\left|\Delta u_{n}+u_{n x x} u_{n y}^{2}+u_{n y y} u_{n x}^{2}-2 u_{n x} u_{n y} u_{n x y}\right|}{\left(1+u_{n x}^{2}+u_{n y}^{2}\right)^{3 / 2}}\left|\Delta u_{n}^{\prime}\right| d \mathbf{x} \\
& \leq \int_{\Omega}\left\{\left|\Delta u_{n}\right|+\left|u_{n x x}\right|+\left|u_{n y y}\right|+\left|u_{n x y}\right|\right\}\left|\Delta u_{n}^{\prime}\right| d \mathbf{x} \\
& \leq c_{1}\left\|\Delta u_{n}\right\|_{L^{2}}^{2}+2\left\|\Delta u_{n}^{\prime}\right\|_{L^{2}}^{2}
\end{aligned}
$$

and consequently (4.4) becomes

$$
\begin{aligned}
& \left\|\nabla u_{n}^{\prime}(t)\right\|_{L^{2}}^{2}+k\left\|\Delta u_{n}(t)\right\|_{L^{2}}^{2}+\gamma\left\|\Delta u_{n}^{\prime}(t)\right\|_{L^{2}}^{2} \\
& \quad \leq c_{2}+c_{3} \int_{0}^{t}\left\{\left\|\Delta u_{n}\right\|_{L^{2}}^{2}+\left\|\Delta u_{n}^{\prime}\right\|_{L^{2}}^{2}\right\} d \tau+2 \int_{0}^{t}\|f\|_{L^{2}}\left\|\Delta u_{n}^{\prime}\right\|_{L^{2}} d \tau \\
& \quad \leq c_{4}+c_{5} \int_{0}^{t}\left\{\left\|\Delta u_{n}\right\|_{L^{2}}^{2}+\left\|\Delta u_{n}^{\prime}\right\|_{L^{2}}^{2}\right\} d \tau .
\end{aligned}
$$

Applying Gronwall's lemma, we have for all $t \in(0, T]$

$$
\left.\begin{array}{l}
\left\|\Delta u_{n}(t)\right\|_{L^{2}} \leq M_{1} \\
\left\|\Delta u_{n}^{\prime}(t)\right\|_{L^{2}} \leq M_{2}
\end{array}\right\}
$$

with $M_{1}$ and $M_{2}$ positive constants independent of $n$. Therefore, it is possible to select from $\left\{u_{n}\right\}$ a subsequence, again denoted by $\left\{u_{n}\right\}$, such that

$$
\lim u_{n}=u
$$

in the weak ${ }^{*}$ topology of $W^{1, \infty}\left(0, T ; H^{2} \cap H_{0}^{1}\right)$ (see [3]).

Regarding the convergence of the nonlinear term, observe that, by Remark 3.3,

$$
\left\|\mathbf{b}\left(\nabla u_{n}\right)-\mathbf{b}(\nabla u)\right\|_{L^{\infty}\left(L^{2}\right)} \leq c\left\|\nabla u_{n}-\nabla u\right\|_{L^{\infty}\left(0, T ; L^{2}\right)}
$$

On the other hand, by compactness arguments, (4.6) implies

$$
\lim _{n \rightarrow+\infty}\left\|\nabla u_{n}-\nabla u\right\|_{L^{\infty}\left(0, T ; L^{2}\right)}=0
$$

so that

$$
\lim _{n \rightarrow+\infty}\left\|\mathbf{b}\left(\nabla u_{n}\right)-\mathbf{b}(\nabla u)\right\|_{L^{\infty}\left(0, T ; L^{2}\right)}=0 .
$$

Let us now prove that the function $u$ defined by (4.6) is a solution. Observe that by (4.6) $u$ satisfies condition a) of Section 3 . Thus, we need to verify condition b). To this aim, let $h \in H^{1}\left(0, T ; H^{2} \cap H_{0}^{1}\right)$ and set

$$
h(t)=\sum_{j=1}^{+\infty} \lambda_{j}(t) g_{j} \quad \text { and } \quad h_{p}(t)=\sum_{j=1}^{p} \lambda_{j}(t) g_{j} .
$$


Multiply (4.1) by $\lambda_{1}, \ldots, \lambda_{p}(p \leq n)$, add and integrate in $[0, t] \cdot(t \in(0, T])$. Bearing in mind that $u_{n}^{\prime}(0)=\beta_{n}$, we have

$$
\begin{aligned}
\int_{0}^{t}\{ & -\left(u_{n}^{\prime}, h_{p}^{\prime}\right)_{L^{2}}-k\left(\Delta u_{n}, h_{p}\right)_{L^{2}}+\gamma\left(\Delta u_{n}^{\prime}, h_{p}^{\prime}\right)_{L^{2}} \\
& \left.+k \rho^{2}\left(\operatorname{divb}\left(\nabla u_{n}\right), h_{p}\right)_{L^{2}}-\left(f, h_{p}\right)_{L^{2}}\right\} d \tau \\
= & -\left(u_{n}^{\prime}(t), h_{p}(t)\right)_{L^{2}}+\left(\beta_{n}, h_{p}(0)\right)_{L^{2}} \\
& +\gamma\left(\Delta u_{n}^{\prime}(t), h_{p}(t)\right)_{L^{2}}-\gamma\left(\Delta \beta_{n}, h_{p}(0)\right)_{L^{2}}
\end{aligned}
$$

Since, for $p$ fixed, by (4.9)

$$
\begin{aligned}
\lim _{n \rightarrow+\infty}\left(\operatorname{divb}\left(\nabla u_{n}\right), h_{p}\right)_{L^{2}\left(0, T ; L^{2}\right)} & =\lim _{n \rightarrow+\infty}\left(\mathbf{b}\left(\nabla u_{n}\right), \nabla h_{p}\right)_{L^{2}\left(0, T ; L^{2}\right)} \\
& =\left(\mathbf{b}(\nabla u), \nabla h_{p}\right)_{L^{2}\left(0, T ; L^{2}\right)} \\
& =\left(\operatorname{div} \mathbf{b}(\nabla u), h_{p}\right)_{L^{2}\left(0, T ; L^{2}\right)}
\end{aligned}
$$

then, if $n \rightarrow+\infty$ in (4.11), we have

$$
\begin{aligned}
\int_{0}^{t}\{ & -\left(u^{\prime}, h_{p}^{\prime}\right)_{L^{2}}-k\left(\Delta u, h_{p}\right)_{L^{2}}+\gamma\left(\Delta u^{\prime}, h_{p}^{\prime}\right)_{L^{2}} \\
& \left.+k \rho^{2}\left(\operatorname{divb}(\nabla u), h_{p}\right)_{L^{2}}-\left(f, h_{p}\right)_{L^{2}}\right\} d \tau \\
= & -\left(u^{\prime}(t), h_{p}(t)\right)_{L^{2}}+\left(\beta, h_{p}(0)\right)_{L^{2}} \\
& +\gamma\left(\Delta u^{\prime}(t), h_{p}(t)\right)_{L^{2}}-\gamma\left(\Delta \beta, h_{p}(0)\right)_{L^{2}}
\end{aligned}
$$

Now, if $p \rightarrow+\infty$ in $(4.12)$, condition b) follows

\section{A uniqueness result}

In the next theorem, the assumptions are the same as in the previous one.

Theorem 5.1. Let $f \in L^{2}(Q), \alpha \in H^{2} \cap H_{0}^{1}$ and $\beta \in H^{2}$. Then, there exists a unique solution of problem (1.3) - (1.5) in $Q$.

Proof. Let $u$ and $v$ be two solutions of problem (1.3) - (1.5) (their existence is guaranteed by Theorem 4.1). Setting $w=u-v$, conditions a) and b) of Section 3 give:

a') $w \in W^{1, \infty}\left(0, T ; H^{2} \cap H_{0}^{1}\right)$ and $w(0)=0$.

b') $w$ satisfies a.e. in $[0, T]$ the equation

$$
\begin{aligned}
\int_{0}^{t}\{- & \left(w^{\prime}, h^{\prime}\right)_{L^{2}}-k(\Delta w, h)_{L^{2}}+\gamma\left(\Delta w^{\prime}, h^{\prime}\right)_{L^{2}} \\
& \left.+k \rho^{2}(\operatorname{divb}(\nabla u)-\operatorname{divb}(\nabla v), h)_{L^{2}}\right\} d \tau \quad\left(h \in H^{1}\left(0, t ; L^{2}\right)\right) \\
= & -\left(w^{\prime}(t), h(t)\right)_{L^{2}}+\gamma\left(\Delta w^{\prime}(t), h(t)\right)_{L^{2}} .
\end{aligned}
$$


Reasoning in a similar way to [5], we construct now a convenient test function. Let $t \in(0, T]$ such that $w^{\prime}(t)$ exists, and let $w_{1}$ and $w^{*}$ be functions defined as

$$
w_{1}(\tau)=\frac{\tau}{t} w^{\prime}(t) \quad(\tau \in[0, t])
$$

and

$$
w^{*}(\tau)= \begin{cases}w^{\prime}(\tau)-w_{1}(\tau) & \text { a.e: in }(0, t] \\ 0 . & \text { for } \tau \in(-\infty, 0] \cup(t,+\infty) .\end{cases}
$$

Setting $h_{\delta}=w_{1}+J_{\delta}^{2} w^{*}$, we observe that $h_{\delta} \in H^{1}\left(0, t ; L^{2}\right)$ (see Lemma 3.1) and we can therefore set $h=h_{\delta}$ in (5.1). So we obtain

$$
\begin{aligned}
\int_{0}^{t}\{ & -\left(w^{\prime}, h_{\delta}^{\prime}\right)_{L^{2}}-k\left(\Delta w, h_{\delta}\right)_{L^{2}}+\gamma\left(\Delta w^{\prime}, h_{\delta}^{\prime}\right)_{L^{2}} \\
& \left.+k \rho^{2}\left(\operatorname{div} \mathbf{b}(\nabla u)-\operatorname{div} \mathbf{b}(\nabla v), h_{\delta}\right)_{L^{2}}\right\} d \tau \\
= & -\left(w^{\prime}(t), h_{\delta}(t)\right)_{L^{2}}+\gamma\left(\Delta w^{\prime}(t), h_{\delta}(t)\right)_{L^{2}}
\end{aligned}
$$

Examine now the behaviour of every term of this last expression when $\delta \rightarrow 0$. As regards the first term of (5.4), observe that

$$
\begin{aligned}
\int_{0}^{t}\left(w^{\prime}, h_{\delta}^{\prime}\right)_{L^{2}} d \tau \\
\quad=\int_{0}^{t}\left(w^{\prime}, w_{1}^{\prime}+\frac{\partial}{\partial \tau} J_{\delta}^{2} w^{*}\right)_{L^{2}} d \tau \\
=\int_{0}^{t}\left(w^{\prime}, w_{1}^{\prime}\right)_{L^{2}}+\int_{0}^{t}\left(w_{1}, \frac{\partial}{\partial \tau} J_{\delta}^{2} w^{*}\right)_{L^{2}} d \tau+\int_{0}^{t}\left(w^{*}, \frac{\partial}{\partial \tau} J_{\delta}^{2} w^{*}\right)_{L^{2}} d \tau \\
=\int_{0}^{t}\left(w_{1}^{\prime}, w^{\prime}-J_{\delta}^{2} w^{*}\right)_{L^{2}} d \tau+\left.\left(w_{1}, J_{\delta}^{2} w^{*}\right)_{L^{2}}\right|_{0} ^{t}+\int_{0}^{t}\left(w^{*}, \frac{\partial}{\partial \tau} J_{\delta}^{2} w^{*}\right)_{L^{2}} d \tau
\end{aligned}
$$

Now, again by Lemma 3.1,

$$
\begin{aligned}
\int_{0}^{t}\left(w^{*}, \frac{\partial}{\partial \tau} J_{\delta}^{2} w^{*}\right)_{L^{2}} d \tau & =\int_{-\infty}^{+\infty}\left(w^{*}, \frac{\partial}{\partial \tau} J_{\delta}^{2} w^{*}\right)_{L^{2}} d \tau \\
& =\int_{-\infty}^{+\infty}\left(J_{\delta} w^{*}, \frac{\partial}{\partial \tau} J_{\delta} w^{*}\right)_{L^{2}} d \tau \\
& =\frac{1}{2} \int_{-\infty}^{+\infty} \frac{d}{d \tau}\left\|J_{\delta} w^{*}\right\|_{L^{2}}^{2} d \tau \\
& =0
\end{aligned}
$$

By (5.6) and being $w_{1}(0)=0,(5.5)$ becomes

$$
\int_{0}^{t}\left(w^{\prime}, h_{\delta}^{\prime}\right)_{L^{2}} d \tau=\int_{0}^{t}\left(w_{1}^{\prime}, w^{\prime}-J_{\delta}^{2} w^{*}\right)_{L^{2}} d \tau+\left(w_{1}(t), J_{\delta}^{2} w^{*}(t)\right)_{L^{2}} .
$$


Letting $\delta \rightarrow 0$ in (5.7), since (see [5])

$$
\left(w_{1}(t), J_{\delta}^{2} w^{*}(t)\right)_{L^{2}}=\left(w^{\prime}(t), J_{\delta}^{2} w^{*}(t)\right)_{L^{2}} \rightarrow 0
$$

it follows that

$$
\begin{aligned}
\lim _{\delta \rightarrow 0} \int_{0}^{t}\left(w^{\prime}, h_{\delta}^{\prime}\right)_{L^{2}} d \tau & =\int_{0}^{t}\left(w_{1}^{\prime}, w^{\prime}-w^{*}\right)_{L^{2}} d \tau \\
& =\int_{0}^{t} \frac{1}{2} \frac{d}{d \tau}\left\|w_{1}\right\|_{L^{2}}^{2} d \tau \\
& =\frac{1}{2}\left\|w_{1}(t)\right\|_{L^{2}}^{2} \\
& =\frac{1}{2}\left\|w^{\prime}(t)\right\|_{L^{2}}^{2} .
\end{aligned}
$$

By similar arguments, concerning the third term of (5.4), we have

$$
\begin{aligned}
& \int_{0}^{t}\left(\Delta w^{\prime}, h_{\delta}^{\prime}\right)_{L^{2}} d \tau \\
& \quad=\int_{0}^{t}\left(\Delta w^{\prime}, w_{1}^{\prime}\right)_{L^{2}} d \tau+\int_{0}^{t}\left(\Delta w_{1}, \frac{\partial}{\partial \tau} J_{\delta}^{2} w^{*}\right)_{L^{2}} d \tau+\int_{0}^{t}\left(\Delta w^{*}, \frac{\partial}{\partial \tau} J_{\delta}^{2} w^{*}\right)_{L^{2}} d \tau
\end{aligned}
$$

Now, keeping in mind that $\left.J_{\delta}^{2} w^{*}(\tau, \mathbf{x})\right|_{\partial \Omega}=0$ for all $\tau$ and by means the well-known properties of $J_{\delta}$, we have

$$
\begin{aligned}
\int_{0}^{t}\left(\Delta w^{*}, \frac{\partial}{\partial \tau} J_{\delta}^{2} w^{*}\right)_{L^{2}} d \tau & =-\int_{0}^{t}\left(\nabla w^{*}, \nabla \frac{\partial}{\partial \tau} J_{\delta}^{2} w^{*}\right)_{L^{2}} d \tau \\
& =\int_{-\infty}^{+\infty} \frac{1}{2} \frac{d}{d \tau}\left\|J_{\delta} \nabla w^{*}\right\|_{L^{2}}^{2} d \tau \\
& =0
\end{aligned}
$$

and consequently, being $\Delta w_{1}(0)=0$,

$$
\begin{aligned}
& \int_{0}^{t}\left(\Delta w^{\prime}, h_{\delta}^{\prime}\right)_{L^{2}} d \tau \\
& \quad=-\int_{0}^{t}\left(\nabla w_{1}^{\prime}, \nabla\left(w^{\prime}-J_{\delta}^{2} w^{*}\right)\right)_{L^{2}} d \tau+\left(\Delta w_{1}(t), J_{\delta}^{2} w^{*}(t)\right)_{L^{2}} .
\end{aligned}
$$

Arguing as in [5], it follows that

$$
\lim _{\delta \rightarrow 0}\left(\Delta w_{1}(t), J_{\delta}^{2} w^{*}(t)\right)_{L^{2}}=0
$$

Hence, by (5.11) and Lemma 3.1,

$$
\lim _{\delta \rightarrow 0} \int_{0}^{t}\left(\Delta w^{\prime}, h_{\delta}^{\prime}\right)_{L^{2}} d \tau=-\int_{0}^{t}\left(\dot{\nabla} w_{1}^{\prime}, \nabla w_{1}\right)_{L^{2}} d \tau=-\frac{1}{2}\left\|\nabla w^{\prime}(t)\right\|_{L^{2}}^{2} .
$$


Concerning the remaining terms of (5.4), we easily deduce (recall Remark 3.3 )

$$
\begin{aligned}
\lim _{\delta \rightarrow 0} \int_{0}^{t}\left(\Delta w, h_{\delta}\right)_{L^{2}} d \tau & =-\frac{1}{2}\|\nabla w(t)\|_{L^{2}}^{2} \\
\lim _{\delta \rightarrow 0} \int_{0}^{t}\left(\operatorname{div} \mathbf{b}(\nabla u)-\operatorname{div} \mathbf{b}(\nabla v), h_{\delta}\right)_{L^{2}} d \tau & =\int_{0}^{t}\left(\operatorname{divb}(\nabla u)-\operatorname{divb}(\nabla v), w^{\prime}\right)_{L^{2}} d \tau \\
& =\int_{0}^{t}\left(\mathbf{b}(\nabla u)-\mathbf{b}(\nabla v), \nabla w^{\prime}\right)_{L^{2}} d \tau \\
\lim _{\delta \rightarrow 0}\left(w^{\prime}(t), h_{\delta}(t)\right)_{L^{2}} & =\left\|w^{\prime}(t)\right\|_{L^{2}}^{2} \\
\lim _{\delta \rightarrow 0}\left(\Delta w^{\prime}(t), h_{\delta}(t)\right)_{L^{2}} & =-\left\|\nabla w^{\prime}(t)\right\|_{L^{2}}^{2}
\end{aligned}
$$

(to deduce the last two limits, refer again to [5]). Hence, by means of (5.8) and (5.12) - (5.16), if $\delta \rightarrow 0$ in (5.4), it follows that

$$
\begin{aligned}
\left\|w^{\prime}(t)\right\|_{L^{2}}^{2} & +k\|\nabla w(t)\|_{L^{2}}^{2}+\gamma\left\|\nabla w^{\prime}(t)\right\|_{L^{2}}^{2} \\
& =2 k \rho^{2} \int_{0}^{t}\left(\mathbf{b}(\nabla u)-\mathbf{b}(\nabla v), \nabla w^{\prime}\right)_{L^{2}} d \tau
\end{aligned}
$$

Recalling Remark 3.2, we get

$$
\begin{aligned}
\left|\int_{0}^{t}\left(\mathbf{b}(\nabla u)-\mathbf{b}(\nabla v), \nabla w^{\prime}\right)_{L^{2}} d \tau\right| & \leq \int_{0}^{t}\left(\int_{\Omega}|\nabla w|\left|\nabla w^{\prime}\right| d \mathbf{x}\right) d \tau \\
& \leq \int_{0}^{t}\left(\frac{1}{2}\|\nabla w\|_{L^{2}}^{2}+\frac{1}{2}\left\|\nabla w^{\prime}\right\|_{L^{2}}^{2}\right) d \tau
\end{aligned}
$$

Using this inequality, from (5.17) we have a.e. in $[0, T]$

$$
k\|\nabla w(t)\|_{L^{2}}^{2}+\gamma\left\|\nabla w^{\prime}(t)\right\|_{L^{2}}^{2} \leq k \rho^{2} \int_{0}^{t}\left(\|\nabla w(\tau)\|_{L^{2}}^{2}+\left\|\nabla w^{\prime}(\tau)\right\|_{L^{2}}^{2}\right) d \tau .
$$

By means of the Gronwall lemma [3: Lemma 0.4.2], we get

$$
\|\nabla w(t)\|_{L^{2}}^{2}+\left\|\nabla w^{\prime}(t)\right\|_{L^{2}}^{2}=0 \quad \text { a.e. in }[0, T]
$$

and consequently $\|w(t)\|_{H_{0}^{1}}=0$ for all $t \in[0, T]$, which implies $w(t)=0$ in $[0, T]$. The assertion follows

\section{References}

[1] Adams, R.: Sobolev Spaces. Orlando: Acad. Press 1975.

[2] Grisvard, P.: Elliptic Problems in Nonsmooth Domains. Boston: Pitman 1985.

[3] Haraux, A.: Nonlinear Evolution Equations. Berlin: Springer Verlag 1981.

[4] Iannelli, A., Prouse, G. and A. Veneziani: Analysis of a nonlinear model of the vibrating string. Nonlin. Diff. Equ. Appl. 3 (1996), 149 - 177.

[5] Prodi, G.: Rassegna di ricerche intorno alle equazioni di Navier-Stokes. Istituto di Matematica dell'Università di Trieste, Quaderno n. 2, 1959. 